\title{
Muros de cortante en estructuras de madera
}

\author{
V. Rubén Ordóñez Candelaria ${ }^{1}$
}

\begin{abstract}
RESUMEN
Este trabajo se realizó con el fin de obtener información sobre el comportamiento estructural de muros bajo cargas laterales, construidos con bastidores de madera y algún forro que proporcione rigidez y resistencia en su plano. Se ensayaron seis forros diferentes considerados como los más apropiados para la construcción con madera en México. Se presentan gráficas de carga deformación para tener idea del comportamiento de los muros de cortante ante las acciones horizontales (acción de sismo o viento). También se proponen valores de diseño para cada uno de los forros, incluyendo información del tipo de conectores utilizados.
\end{abstract}

PALABRAS CLAVES

Muros de cortante, forro estructural, bastidor, aplanado de mortero, cargas laterales.

\begin{abstract}
Structural performance of shear walls was studied in this project, using the standard wall frame and six different sheathings for structural uses in walls under racking loads. The sheathings tested were those deemed the most appropiate for the wood construction industry in Mexico. Curves of load deflection for racking test are presented to give an idea of the behavior of shear walls against racking loads (resulting from earthquake or wind). Also included are proposed design vales for each kind of sheathing and connector used.
\end{abstract}

\section{KEYWORDS}

Shear walls, structural sheathing, wall frame, cement mortar, racking loads.

1 Investigador. Departamento de Productos Forestales y Conservación de Bosques. Instituto de Ecología, A.C. Apdo. Postal 63. Xalapa, Ver., 91000, México.

Manuscrito recibido para su publicación el 12 de Julio de 1994. 


\section{INTRODUCCION}

Todas las estructuras están sujetas a la acción de fuerzas laterales generadas por la acción del viento y en algunas zonas geográficas pueden estar sujetas a fuerzas laterales provocadas por el movimiento del suelo durante la acción de un sismo. Otros tipos de cargas laterales que puedan actuar en las estructuras son las presiones de tierra o explosiones. En este trabajo se presenta una propuesta para el diseño de los sistemas construidos con madera para resistir los diferentes tipos de cargas laterales.

Los sistemas constructivos que resisten las cargas laterales en una estructura de madera se muestran en la figura 1; cada uno de los subsistemas de muros, pisos y techos forrados de duela, contrachapados $u$ otro tipo de tableros, se debe diseñar de tal manera que pueda resistir cargas laterales además de las verticales, en este trabajo solo se estudia el muro bajo cargas laterales en su plano. A los elementos verticales se les denominan "muros de cortante" y a los horizontales "diafragmas". Un aspecto importante es el funcionamiento correcto de estos sistemas, lo constituyen las conexiones necesarias para asegurar que éstos actúen como unidades integrales y que esas unidades se interconecten en la construcción como un todo para asegurar el funcionamiento aceptable de la estructura completa.

El uso de la madera en México para
la construcción de estructuras permanentes se está incrementando cada vez más, y una aplicación importante es en la vivienda. Para el diseño estructural de las construcciones se requiere información técnica puesto que el uso óptimo de los materiales se da en la medida en que la información es más confiable. Los muros son elementos que representan un volumen importante de la construcción y de los cuales depende la seguridad de la estructura cuando actúa sobre ella fuerzas horizontales (Fig.1). En México se ha empezado a generar información sobre la rigidez y resistencia de estos elementos utilizando diversos materiales de forro como contrachapados 0 similares, duela, aplanados, etc.

En este trabajo nos enfocaremos a la revisión de los muros de cortante, de los cuales haremos un análisis de diversas soluciones, revisaremos el comportamiento de algunos de ellos y con los datos de las pruebas propondremos valores de resistencia para el diseño o revisión de muros de cortante. Las soluciones que planteamos son las que se pueden construir con relativa facilidad utilizando los materiales disponibles en el país.

\section{TIPOS DE SOLUCIONES}

Las soluciones de "muros de cortante" que revisaremos en este inciso son las que cuentan como elemento básico un bastidor sobre el cual se colocan los diferentes materiales de recubrimiento que sirven para impartirle resistencia y rigidez al muro en su plano. Las características del bastidor se muestran en la figura 2.

La cubierta (o forro) de los muros de cortante puede ser de muy diversos productos a base de madera $u$ otro material. Entre los más comunes se encuentran los tableros de contrachapados u otro tipo de tableros como son los estructurales de partículas; se utiliza también duela machihembrada y aplanado con mortero de cemento-arena reforzado con malla de alambre. Cada uno de éstos se coloca sobre el bastidor siguiendo las recomendaciones básicas de la figura 3 , sin que sea esta la única forma de utilizarlos, ya que la resistencia de los 


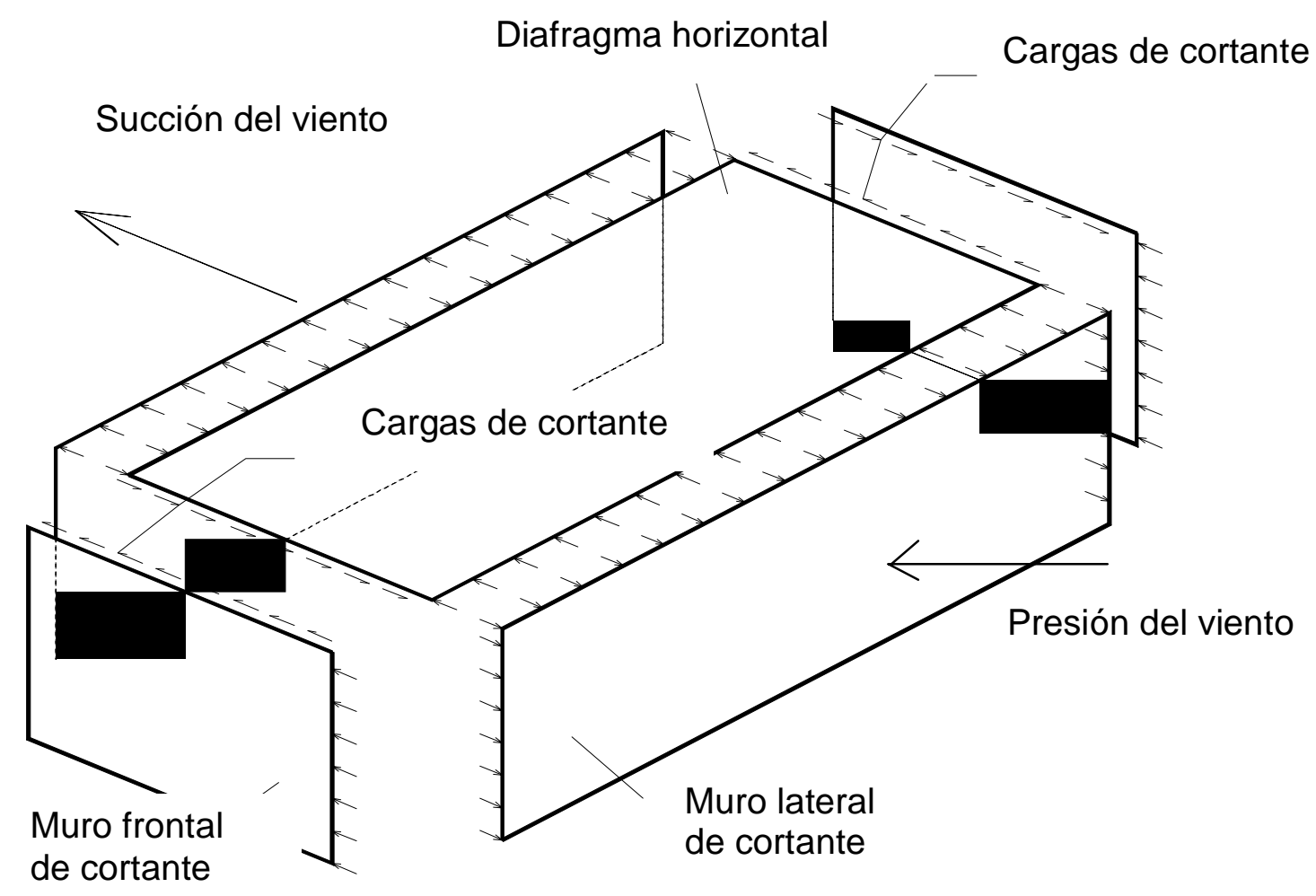

Figura 1 Muros de cortante y diafragmas horizontales de una estructura de madera

paneles depende del tipo de conectores y de la separación entre ellos, por lo tanto, si se requiere un elemento con mayor resistencia se modificaría la disposición y tamaño de los conectores.

Se estudiaron experimentalmente muros con seis recubrimientos diferentes, considerados como los de mayores posibilidades de uso en la construcción de viviendas, que son las siguientes:

a. Contrachapado de madera de pino de $9 \mathrm{~mm}$ de espesor, en tableros de 122 $\mathrm{x} 244 \mathrm{~cm}$. b. Contrachapado de madera de pino de $12 \mathrm{~mm}$ de espesor, en tableros de $122 \times 244 \mathrm{~cm}$.

c. Duela machihembrada de madera de pino de 19 × $87 \mathrm{~mm}$ de sección, colocada horizontalmente.

d. Duela machihembrada de madera de pino de 19 × $87 \mathrm{~mm}$ de sección, colocada diagonalmente, sujeta a cargas de tensión

e. Duela machihembrada de madera de pino de 19 × $87 \mathrm{~mm}$ de sección, colocada diagonalmente, sujeta a cargas de compresión.

f. Forro con aplanado de mortero cemento:arena de $15 \mathrm{~mm}$ de espesor y reforzado con malla metálica (tela de gallinero). 


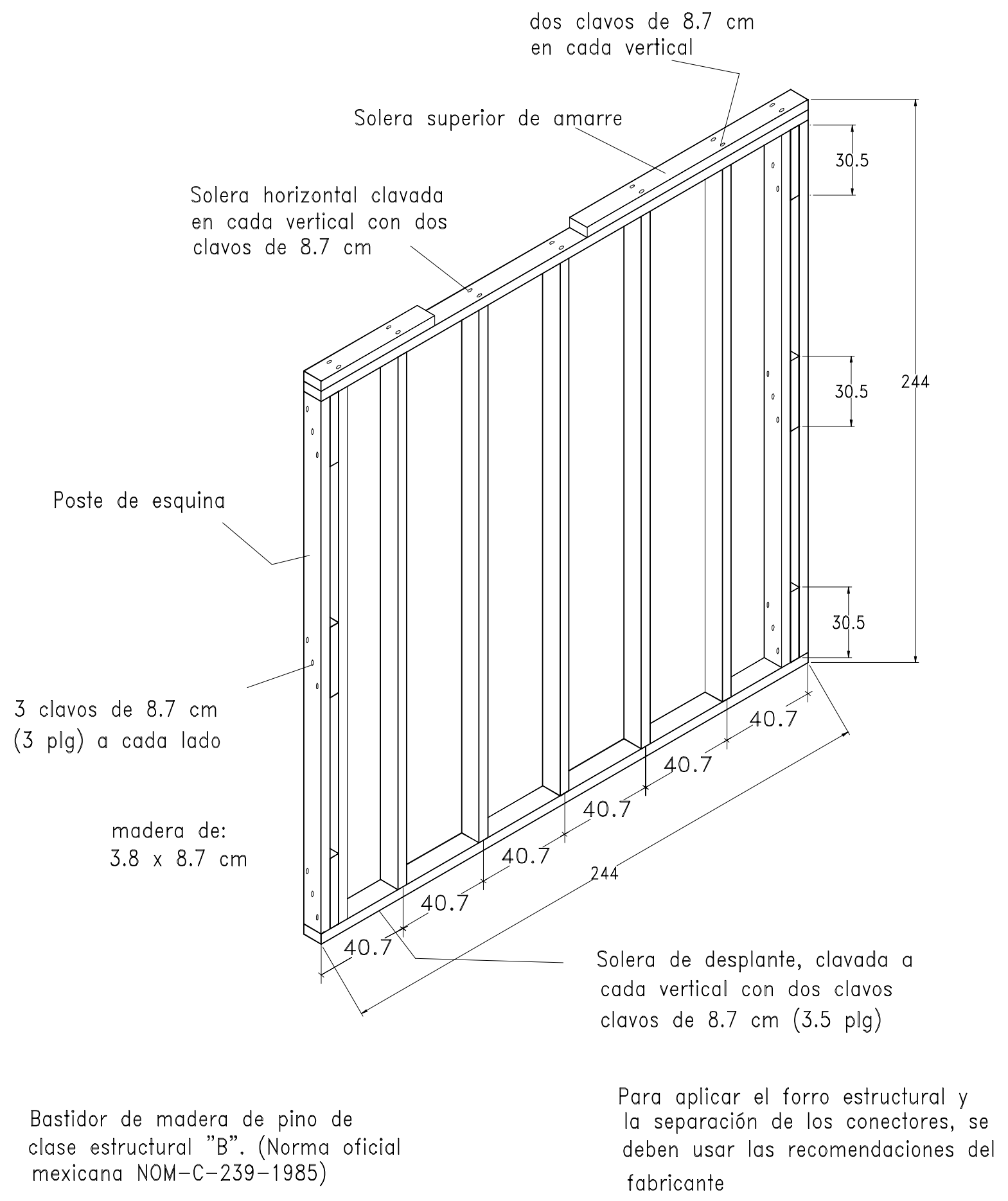

Figura 2. Bastidor de madera para construir muros de cortante colocándole diversos tipos de forros estructurales 


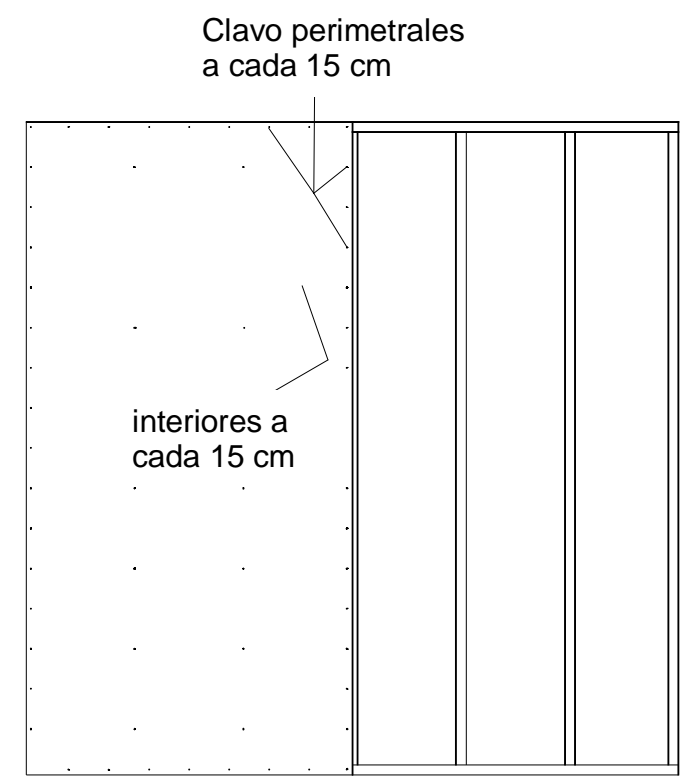

Clavo perimetrales a cada $15 \mathrm{~cm}$

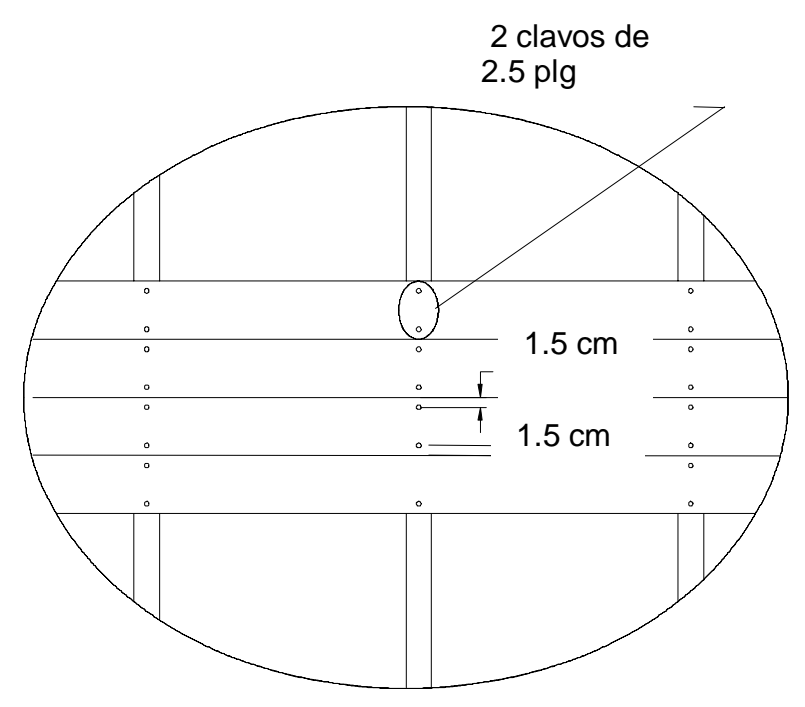

b) duela machihembrada de $19 \mathrm{~mm}$ de espesor

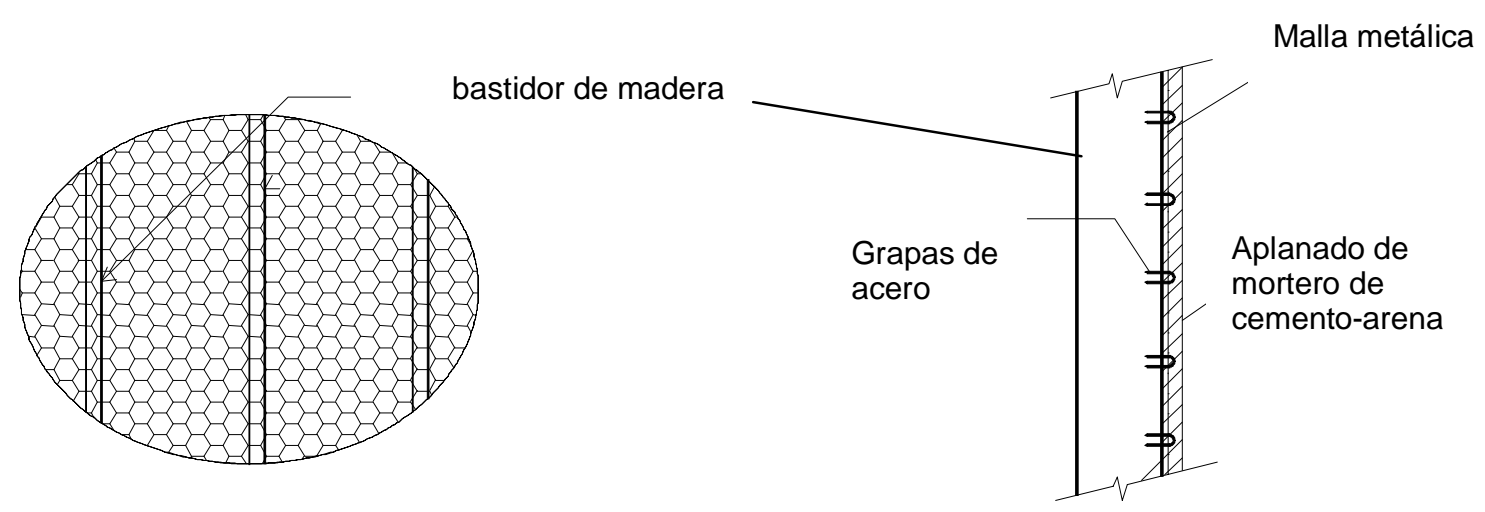

c) Aplanado de mortero de cemento:arena, reforzado con malla metálica (tela de gallinero)

Figura 3. Ejemplos de forro para usar en muros de cortante 
En la tabla 1 se presenta una descripción de los tableros ensayados para cada uno de los diferentes forros. Los bastidores que se utilizaron para la fabricación de los muros cumplen con lo especificado en las normas de las ASTM E72-80(1981),y utilizando madera de pino de clase estructural "B" clasificada conforme a la NOM-C-239-1985 (DGN, 1985) en condición seca $(\mathrm{CH}=18 \%)$.

Los tableros de recubrimiento (forro) se montaron sobre los bastidores apegándose a las recomendaciones de algunos manuales de construcción, como Sherwood and Stroh (1989). Para el caso de los tableros de cemento se propuso el procedimiento, ya que no se encontró información publicada al respecto; el esfuerzo de malla metálica se fijó al bastidor utilizado grapas metálicas galvanizadas y posteriormente se aplicó el aplanado. En la tabla 1 se describen los conectores y su separación para los demás foros y en la figura 3 se observan los detalles de aplicación como ya se indicó.

Tabla 1. Características de los muros ensayados

\begin{tabular}{|c|c|c|c|c|}
\hline \multirow{2}{*}{\multicolumn{2}{|c|}{ TIPO DE FORRO }} & \multirow[t]{2}{*}{ CONECTORES } & \multicolumn{2}{|c|}{$\begin{array}{c}\text { SEPARACION DE } \\
\text { CONECTORES, } \\
\mathrm{cm}\end{array}$} \\
\hline & & & borde & int \\
\hline & $\begin{array}{l}\text { Contrachapado de madera de pino de } 9 \\
\text { mm de espesor. }\end{array}$ & $\begin{array}{c}\text { Clavos de } 51 \mathrm{~mm} \\
(2 \text { pulg })\end{array}$ & 15 & 30 \\
\hline b) & $\begin{array}{l}\text { Contrachapado de madera de pino de } 12 \\
\text { mm de espesor. }\end{array}$ & $\begin{array}{c}\text { Clavos de } 63.5 \mathrm{~mm} \\
(2.5 \text { pulg })\end{array}$ & 15 & 30 \\
\hline c) & $\begin{array}{l}\text { Duela machihembrada de pino, colocada } \\
\text { en forma horizontal. }\end{array}$ & $\begin{array}{c}\text { Clavos de } 63.5 \mathrm{~mm} \\
(2.5 \text { pulg })\end{array}$ & (1) & (1) \\
\hline d) & $\begin{array}{l}\text { Duela machihembrada de pino, colocada } \\
\text { en diagonal sujeta a cargas de tensión. }\end{array}$ & $\begin{array}{c}\text { Clavos de } 63.5 \mathrm{~mm} \\
(2.5 \text { pulg })\end{array}$ & (1) & (1) \\
\hline e) & $\begin{array}{l}\text { Duela machihembrada de pino, colocada } \\
\text { en diagonal sujeta a cargas de } \\
\text { compresión. }\end{array}$ & $\begin{array}{l}\text { Clavos de } 63.5 \mathrm{~mm} \\
(2.5 \text { pulg })\end{array}$ & (1) & (1) \\
\hline & $\begin{array}{l}\text { Aplanado de cemento-arena en } \\
\text { proporción de } 1: 3 \text {, de } 15 \mathrm{~mm} \text { de espesor, } \\
\text { reforzado con malla de alambre. }\end{array}$ & $\begin{array}{l}\text { Grapas } \\
\text { galvanizadas(2) }\end{array}$ & 20 & 30 \\
\hline
\end{tabular}

1 Se usaron dos clavos en el cruce de cada duela con los verticales del bastidor

2 Grapas iguales a las que se utilizan en las cercas con alambre de púas 


\section{DETERMINACIÓN DE LA RESISTENCIA Y RIGIDEZ}

\section{Procedimiento de pruebas}

La figura 4 presenta un esquema del montaje de las pruebas que se realizaron para definir las resistencia y rigidez de los muros. En esta se muestra la forma de aplicación de la carga, y los puntos donde se midieron las deformaciones $y$ desplazamientos. La carga se aplicó por medio de un cilindro hidráulico de 25 toneladas de capacidad tomando las lecturas de cargas con un manómetro calibrado con la Máquina Universal con una resolución de $50 \mathrm{~kg}$. Las deformaciones y desplazamientos del muro se obtuvieron por medio de indicadores de carátula; en el punto 1 se tiene la deformación total del muro $\left(d_{1}\right)$, en el punto 2 el posible desplazamiento $\left(d_{2}\right)$ de la base del muro y en el punto 3 el desplazamiento vertical $\left(\mathrm{d}_{3}\right)$ debido a posibles rotaciones del muro. La deformación por cortante (d) del muro se obtiene con la siguiente expresión:

$$
d=d_{1}-d_{2}-d_{3}
$$

El montaje de las pruebas se efectuó conforme a la norma ASTM E72-80 (1981). El Procedimiento de carga empleado consistió de cuatro etapas; en la primera el muro se cargó de 0 a $400 \mathrm{~kg}$ con un rango de velocidad tal que se alcanzaran $400 \mathrm{~kg}$ en no menos de 2 minutos; en este punto se descargó el espécimen permitido se recuperación y registrando la deformación permanente causada por este valor de carga; en la segunda y tercera etapa se alcanzaron valores de cargas de $800 \mathrm{~kg}$ y $1200 \mathrm{~kg}$ respectivamente, con la misma velocidad de carga que la aplicada en la etapa 1; también se descargó el muro y se registraron las deformaciones permanentes en cada una de ellas. Finalmente, en la última etapa se aplicó carga hasta que fallara el muro o cuando la deformación horizontal del mismo superara los $10 \mathrm{~cm}$. En cada etapa de carga se registraron las deformaciones correspondientes a intervalos de $100 \mathrm{~kg}$. Con el fin de observar el comportamiento de cada uno de los diferentes tipos de recubrimiento se obtuvo la fórmula de una curva de ajuste de los valores de las pruebas tomando únicamente los datos de prueba como si se tratara de una carga continua desde cero hasta la carga máxima; los resultados de las pruebas se muestran en las gráficas de la 5 a 10.

La resistencia y rigidez de los muros se calcularon conforme a lo establecido en la norma ASTM E564-76 (1976). La resistencia simplemente consiste en dividir la carga máxima por la longitud del espécimen, esto es:

$$
S_{u}=\frac{P_{u}}{b}
$$

Donde:

$\mathrm{S}_{\mathrm{u}}=$ Resistencia última a cortante del diafragma, $\mathrm{kg} / \mathrm{m}$

$\mathrm{P}_{\mathrm{u}}=$ Carga máxima soportada por el diafragma, $\mathrm{kg}$

$\mathrm{b}=$ Longitud del muro de cortante medido paralelamente a la línea de acción de la carga, $m$

La rigidez a cortante de los diafragmas se calculó con la siguiente expresión:

$$
G^{\prime}=\frac{P a}{b d}
$$




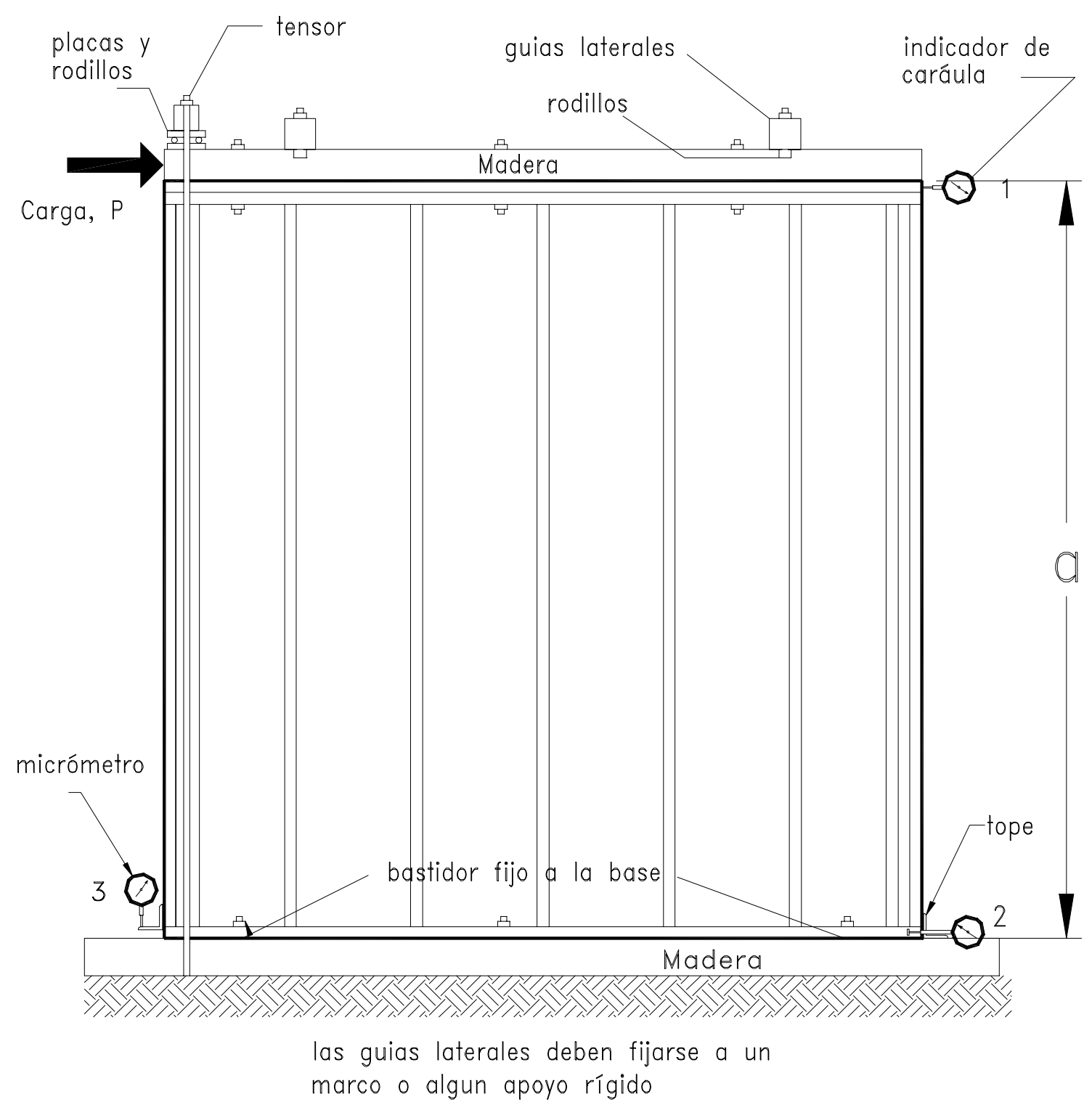

Figura 4. Montaje de las pruebas de muros sujetos a cargas horizontales 
Donde:

$$
\begin{aligned}
G^{\prime}= & \begin{array}{l}
\text { Rigidez a cortante del muro } \\
\text { obtenida de la prueba, } \\
\text { incluyendo la deformación del } \\
\text { sistema de conexiones, } \mathrm{kg} / \mathrm{m}
\end{array} \\
\mathrm{P}= & \begin{array}{l}
\text { Carga concentrada en el extremo } \\
\text { del muro. Aproximadamente } 1 / 3 \\
\text { de la carga última }\left(\mathrm{P}_{\mathrm{u}}\right), \mathrm{kg}
\end{array} \\
\mathrm{a}= & \begin{array}{l}
\text { Altura del muro de cortante como } \\
\text { voladizo, } \mathrm{m}
\end{array} \\
\mathrm{b}= & \begin{array}{l}
\text { Longitud del muro de cortante } \\
\text { medido paralelamente a la línea de }
\end{array} \\
& \begin{array}{l}
\text { acción de la carga, m } \\
\mathrm{d}_{1}-\mathrm{d}_{2}-\mathrm{d}_{3} . \text { Deformación por }
\end{array} \\
\mathrm{d}= & \begin{array}{l}
\text { cortante del muro (incluye la } \\
\text { deformación por cortante del } \\
\text { recubrimiento y la deformación de }
\end{array} \\
& \text { los conectores), } \mathrm{m}
\end{aligned}
$$

\section{RESULTADOS}

Los resultados se presentan en la Tabla 2, y consisten de los valores promedio de rigidez y resistencia de las pruebas que se hicieron con cada uno de los forros. En las figuras 5 a la 10 se observa la curva de carga-deformación de los muros de cortante.

Resulta importante hacer notar que el valor de la resistencia de los diafragmas cubre un rango amplio, que va desde la resistencia de la duela horizontal (Fig. 7) hasta el otro extremo representado por la duela inclinada sujeta a cargas de tensión (Fig. 8) con una resistencia diez veces superior a la de la duela horizontal. Con el uso de duelas colocadas horizontalmente, el muro tiene la desventaja de grandes deformaciones bajo las cargas horizontales con los problemas que estos generan. Cambiando la posición del mismo material, como el caso de la duela inclinada a tensión, se mejora enormemente los valores de resistencia, esto debido al número de duelas de la franja central, que actúan como diagonales en tensión, así como el número de conectores que interactúan para obtener un diafragma con muy alta resistencia. No ocurre lo mismo con la duela inclinada en compresión (Fig. 9) porque las duelas de la franja central generan cargas sobre los elementos verticales de los extremos del bastidor, generando en algunos casos la falla de estos. $Y$ en otros, los clavos ubicados en los extremos de las duelas agotan su capacidad. Pero debe tenerse en cuenta que la acción de las fuerzas sísmicas es alternada por lo que la duela inclinada estaría sujeta a cargas de tensión y compresión cíclicamente.

La falla de los muros con contrachapado (Fig. 3 y 6) está gobernada por la deformación de los conectores en las esquinas con las mayores concentraciones de esfuerzos. No existe una diferencia muy marcada entre ambos espesores como la que hay entre los otros tipos de forro, pero se ve que son de los paneles con valores altos de resistencia.

Los muros con aplanado de cemento (Fig. 10) su resistencia es de valor intermedio; se observó que depende directamente de la resistencia del alambre de la tela de gallinero, puesto que se notó en todos los muros ensayados que el tablero quedó prácticamente íntegro y los conectores rompieron el alambre de la malla. Es de esperar que una mayor cantidad de conectores en el perímetro del tablero incremente la resistencia de este tipo de forro; esto se deduce al analizar el tipo de falla. Otra posibilidad para aumentar la resistencia parece ser el incremento en el diámetro del alambre o algún tipo de refuerzo perimetral en la malla. Los muros con este tipo de forro resultaron ser los de mayor rigidez, sin embargo tienen un comportamiento frágil con baja absorción de energía. Este comportamiento no es recomendable cuando la estructura es solicitada por un 
sismo o viento. Es posible cambiar este tipo de comportamiento haciendo modificaciones al sistema constructivo, para obtener un comportamiento con mayor ductilidad en este tipo de muros como el que presenta una de las curvas que se extiende mas a la derecha que las otras dos. Para lo cual es necesario realizar pruebas variando los factores que influyen en el comportamiento, tales como tipo y diámetro de la malla, separación de conectores, etc.

Un material que se confirma como adecuado para construir edificios que puedan enfrentar con éxito a los sismos o vientos es la madera contrachapada ya que la energía que puede absorber es mayor que el de los demás materiales como se puede observar en las curvas de carga-deformación.

En la tabla 2 se presenta una propuesta de valores de diseño para cada uno de los forros estudiados, que se obtuvieron aplicando un factor de seguridad de $3(F S=3)$, en un caso, y en el otro limitando los desplazamientos horizontales de entrepiso a 1/240 de su altura, (DDF, 1987).

Tabla 2. Valores promedio y mínimo resistencia y rigidez de los forros estudiados

\begin{tabular}{|c|c|c|c|c|c|}
\hline \multirow{2}{*}{\multicolumn{2}{|c|}{ TIPO DE MURO }} & \multirow[t]{2}{*}{$\begin{array}{l}\text { RESISTENCIA } \\
\mathrm{kg} / \mathrm{m}\end{array}$} & \multirow[t]{2}{*}{$\begin{array}{c}\text { RIGIDEZ } \\
\mathrm{kg} / \mathrm{m}\end{array}$} & \multicolumn{2}{|c|}{$\begin{array}{l}\text { RESISTENCIA DE } \\
\text { DISEÑO } \\
\mathbf{k g} / \mathbf{m}\end{array}$} \\
\hline & & & & $F S=3$ & $H / 240$ \\
\hline & $\begin{array}{l}\text { Con forro de contrachapado de } 9 \\
\mathrm{~mm} \text { de espesor ( } 5 \text { pruebas). }\end{array}$ & 992 & 115,600 & 331 & 394 \\
\hline & $\begin{array}{l}\text { Con forro de contrachapado de } 12 \\
\mathrm{~mm} \text { de espesor ( } 5 \text { pruebas). }\end{array}$ & 1,183 & 140,200 & 394 & 465 \\
\hline & $\begin{array}{l}\text { Con forro de duela } \\
\text { machihembrada de pino colocada } \\
\text { horizontalmente (4 pruebas). }\end{array}$ & 203 & 7,000 & 68 & 80 \\
\hline & $\begin{array}{l}\text { Con forro de } \text { duela } \\
\text { machihembrada de pino en } \\
\text { diagonal y sujeta a cargas de } \\
\text { tensión (4 pruebas). }\end{array}$ & 2,219 & 122,500 & 740 & 510 \\
\hline & $\begin{array}{l}\text { Con forro de duela } \\
\text { machihembrada de pino en } \\
\text { diagonal y sujeta a cargas de } \\
\text { compresión ( } 4 \text { pruebas). }\end{array}$ & 755 & 80,400 & 252 & 287 \\
\hline & $\begin{array}{l}\text { Con aplanado de cemento:arena } \\
\text { en proporción de } 1: 3 \text { y refuerzo } \\
\text { con malla metálica ( } 3 \text { pruebas). }\end{array}$ & 785 & 246,800 & 262 & $388(1)$ \\
\hline
\end{tabular}

1 Este valor de diseño corresponde a una deformación igual a la mitad de la utilizada para los demás forros. Esto debido a su alta rigidez de este tipo de material. 
Tabla 3. Valores de diseño para muros de cortante con algunos espesores de contrachapados norteamericanos ( APA, 1987)

\begin{tabular}{|l|c|c|c|}
\hline \multicolumn{1}{|c|}{ TIPO DE FORRO } & $\begin{array}{c}\text { ESPESOR } \\
\mathbf{m m}\end{array}$ & $\begin{array}{c}\text { DIMENSIONES DE } \\
\text { LOS CLAVOS (1) } \\
\text { pulg (mm) }\end{array}$ & $\begin{array}{c}\text { RESISTENCIA } \\
\mathbf{~ k g / m}\end{array}$ \\
\hline \multirow{2}{*}{$\begin{array}{l}\text { APA structural I rated } \\
\text { sheathing }\end{array}$} & 9.5 & $2(50)$ & 342 \\
\cline { 2 - 4 } & 12 & $\begin{array}{c}2.5(63) \\
3(75)\end{array}$ & $\begin{array}{c}\mathbf{m} \\
\text { APA rated sheathing }\end{array}$ \\
\cline { 2 - 4 } & 9.5 & $2.5(63)$ & 327 \\
\hline & 12 & $\begin{array}{c}2.5(63) \\
3(75)\end{array}$ & 387 \\
\hline
\end{tabular}

1 Los clavos se colocan a cada $15 \mathrm{~cm}$ en los bordes del panel, sobre un bastidos similar al que se usó en las pruebas

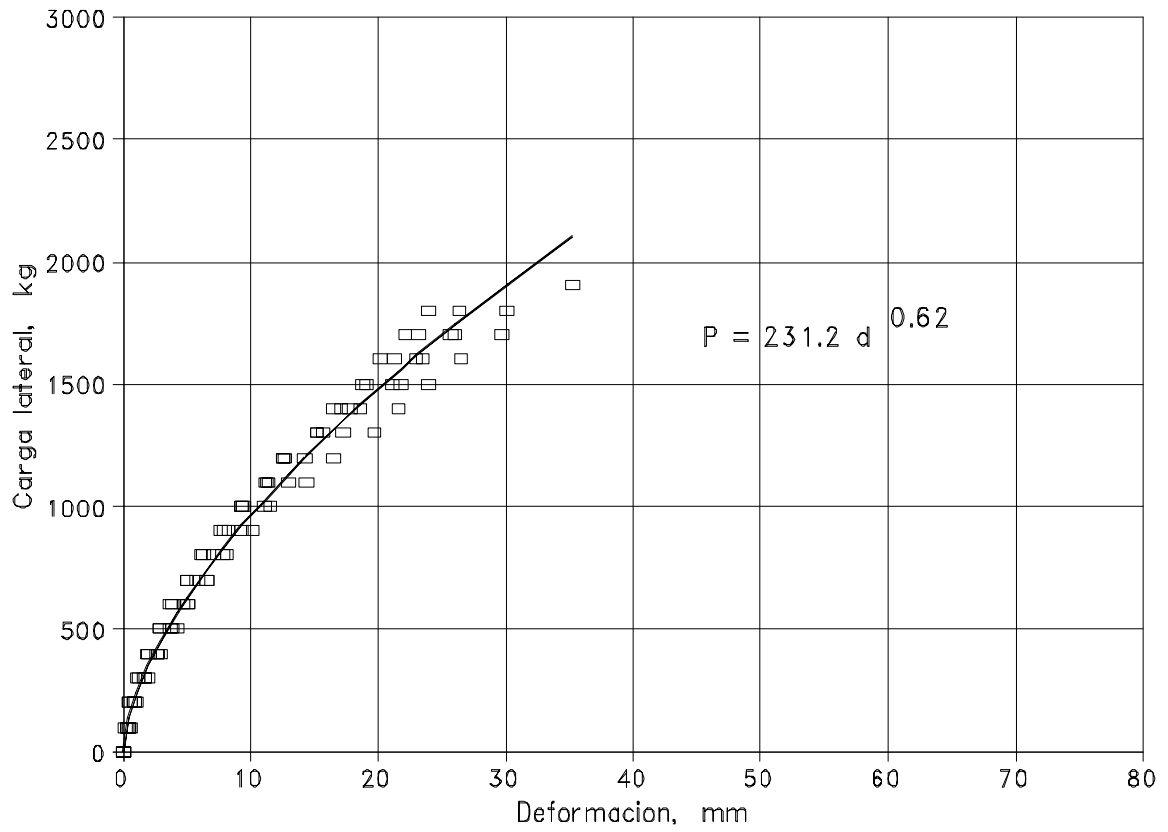

Figura 5. Resultados de muros con forro de madera contrachapada de $9 \mathrm{~mm}$ 


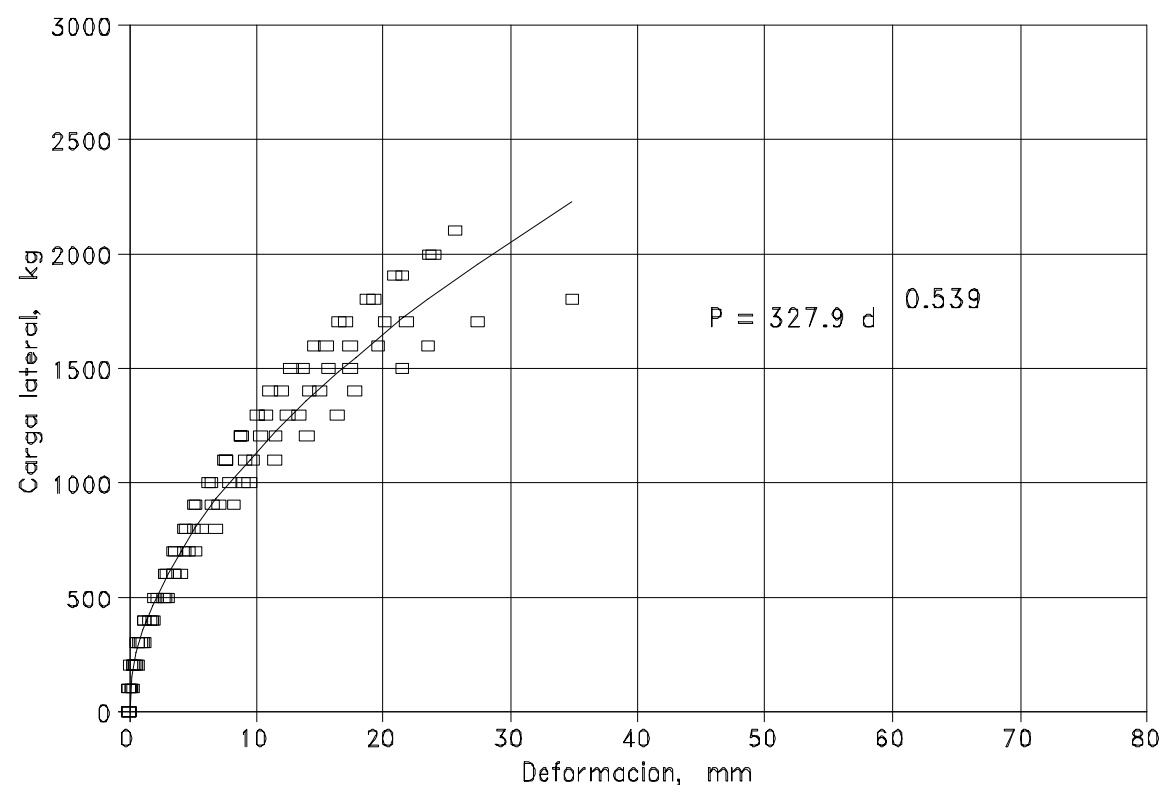

Figura 6. Resultados de muros con forro de madera contrachapada de $12 \mathrm{~mm}$

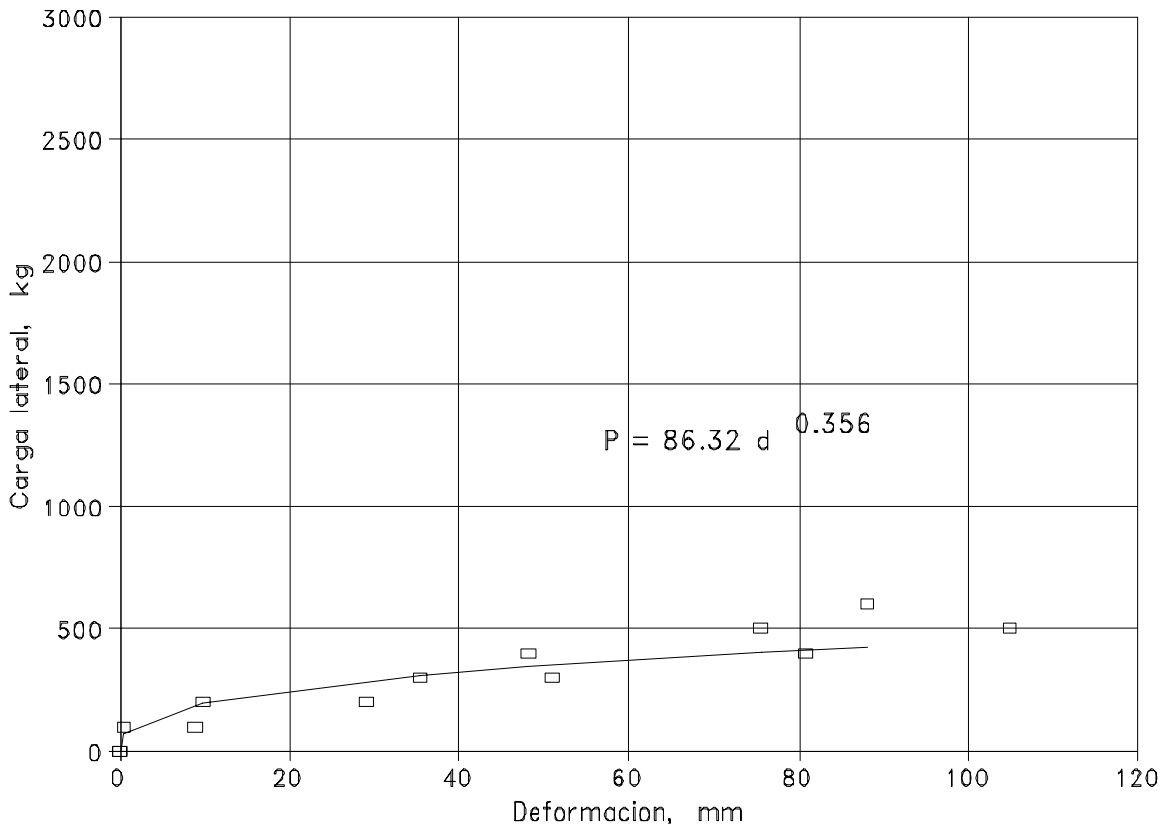

Figura 7. Resultados de muros con forro de duela horizontal de $19 \mathrm{~mm}$ 


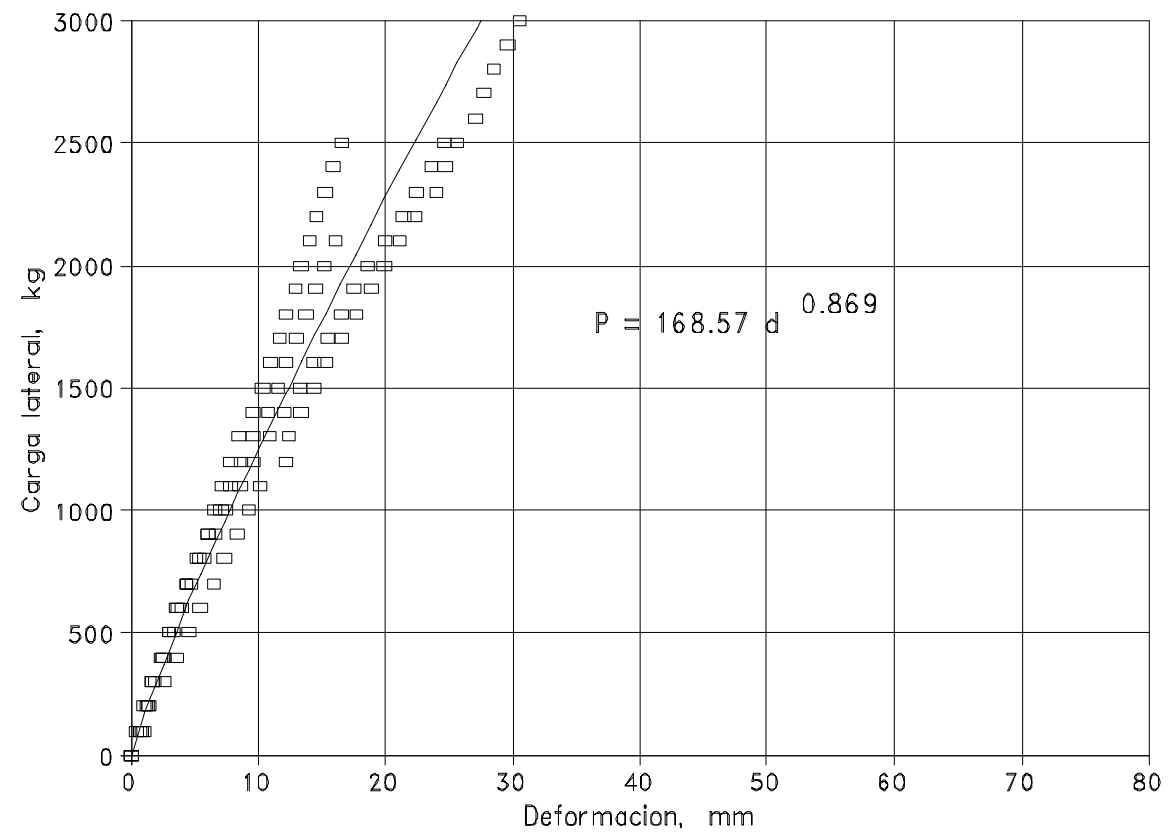

Figura 8. Resultados de muros con forro de duela inclinada de $19 \mathrm{~mm}$, sujeta a tensión

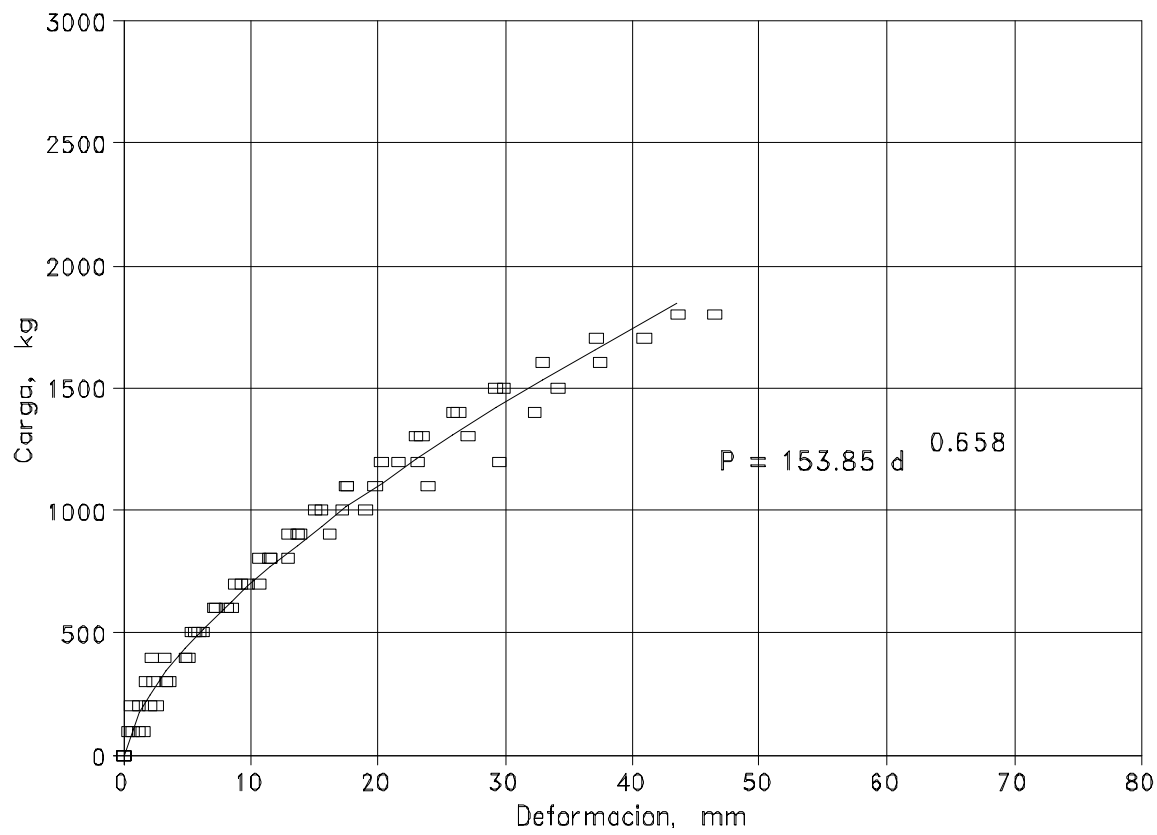

Figura 9. resultados de muros con forro de duela inclinada de $19 \mathrm{~mm}$, sujeta a compresión 


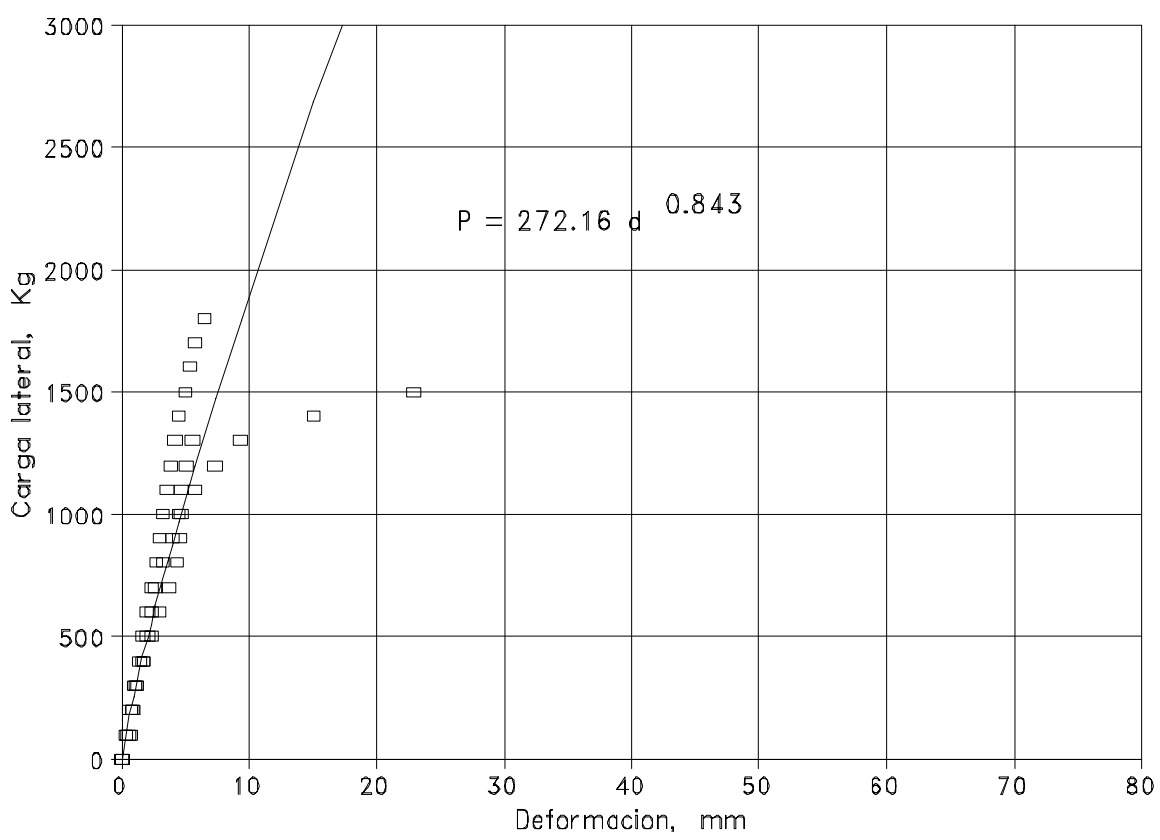

Figura 10. Resultados de muros con aplanado de cemento de $15 \mathrm{~mm}$ de espesor reforzado con tela de gallinero

\section{RECONOCIMIENTOS}

Esta investigación se efectuó con el apoyo financiero del Consejo Nacional de Ciencia y Tecnología. Ref. P.127CCOT90/ 4901, "Determinación de la rigidez y resistencia de muros de cortante con ocho recubrimientos diferentes" y recursos fiscales asignados al Departamento de Productos Forestales y Conservación de Bosques con la clave 902-13.

\section{REFERENCIAS}

American Society for Testing and Materials. 1976. Standard Methods of Static Load Tests for Shear Resistance of Framed Walls for Buildings. ASTM E564-76. ASTM, Philadelphia, $\mathrm{Pa}$.

American Society for Testing and Materials. 1981. Standard Methods of Conducting Strength Tests of Panels for Building Construction. ASTM E7280. ASTM, Philadelphia, Pa.

American Plywood Association. 1987. APA Design, Construction Guide. Diaphragms. Tacoma, Wa.

Departamento del Distrito Federal. 1987. Reglamento de Construcciones para el Distrito Federal. Gaceta Oficial DDF, Quinta Epoca No. 9, México D.F. 1987.100 pp.

Dirección General de Normas. 1985. NOM-C-239-1985. Calificación visual para madera de pino de usos estructurales. SECOFI. México D.F. $13 \mathrm{pp}$

Sherwood, G. E. y R. C. Stroh. 1989. Wood Frame House Construction. USDA, Forest Service. Agricultura Handbook 73. 